\title{
Efficacy And Safety of Canakinumab As A Second- Line Biologic After Tocilizumab Treatment Failure in Children With Systemic Juvenile Idiopathic Arthritis: A Single-Center Cohort Study Using Routinely Collected Health Data
}

Ekaterina Alexeeva ( $\nabla$ alekatya@yandex.ru )

National Medical Research Centre for Children's Health

Elizaveta Krekhova

IM Sechenov First Moscow State Medical University (Sechenovskiy University)

Tatyana Dvoryakovskaya

National Medical Research Centre for Children's Health

Ksenia Isaeva

National Medical Research Centre for Children's Health

Aleksandra Chomakhidze

National Medical Research Centre for Children's Health

Evgeniya Chistyakova

National Medical Research Centre for Children's Health

Olga Lomakina

National Medical Research Centre for Children's Health

Rina Denisova

National Medical Research Centre for Children's Health

Anna Mamutova

National Medical Research Centre for Children's Health

Anna Fetisova

National Medical Research Centre for Children's Health

Marina Gautier

National Medical Research Centre for Children's Health

Dariya Vankova

National Medical Research Centre for Children's Health

Ivan Kriulin

National Medical Research Centre for Children's Health

Ruslan Saygitov

Association of Paediatric Rheumatologists 


\section{Research Article}

Keywords: systemic juvenile idiopathic arthritis, tocilizumab, canakinumab, second line biologic, switching

Posted Date: December 17th, 2021

DOI: https://doi.org/10.21203/rs.3.rs-1174736/v1

License: (c) (i) This work is licensed under a Creative Commons Attribution 4.0 International License.

Read Full License 


\section{Abstract}

Background: A significant number of systemic juvenile idiopathic arthritis (sJIA) patients discontinue biologic disease-modifying antirheumatic drugs (DMARDs) due to lack of efficacy or safety concerns. Studies of biologic therapy switch regimens in SJIA are required.

Methods: Patients with sJIA who switched from tocilizumab (due to lack of efficacy or safety) to canakinumab (4 mg/kg every 4 weeks) and were hospitalized at the rheumatology department from August 2012 to July 2020 were included. Primary efficacy outcomes were $30 \%$ or greater improvement based on the pediatric criteria of the American College of Rheumatology (ACR30), achievement of inactive disease (JADAS-71 $=0$ ) and clinical remission (ACR sJIA clinical inactive disease criteria). Follow-up was 12 months or the closest time point (not less than 6 and not more than 18 months). Data were extracted from outpatient medical records.

Results: During the study period, 46 patients with SJIA switched from tocilizumab to canakinumab. Median age at canakinumab initiation was 8.2 (interquartile range $4.0 ; 12.9$ ) years, and median SJIA duration was $1.8(0.8 ; 5.8)$ years; $37(80 \%)$ patients received at least one non-biologic DMARD (oral corticosteroids, methotrexate and/or cyclosporine A). Study outcomes were followed up in 45 patients (one patient did not attend the follow-up for an unknown reason); median follow-up was $359(282 ; 404)$ days. During the follow-up, canakinumab was discontinued in one patient (due to tuberculosis detection) and the dose was reduced or the injection interval increased in four (9\%) patients. In total, 27 (60\%) patients continued to receive at least one non-biologic DMARD. Improvement according to the ACR30 criteria was achieved in 43 patients (96\%; 95\% confidence interval, 85-99), inactive disease in 42 (93\%; 82-98), and clinical remission in 37 (82\%; 69-91); after adjustment for actual time-at-risk, the rates were 83,85 and 73 events per 100 person-years, respectively. During follow-up, 23 adverse events (most frequently infections) were reported in 19/45 (42\%) patients; $5 / 45$ (11\%) patients developed macrophage activation syndrome, with a favorable outcome in all cases.

Conclusions: Short-term (about 12 months) canakinumab therapy was found to be potentially effective as second-line biologic therapy after discontinuation of tocilizumab in patients with sJIA.

Trial registration: CACZ885G2301E1 (G2301; NCT00891046 registered on April 29, 2009) and CACZ885G2306 (G2306; NCT02296424 registered on November 20, 2014).

\section{Background}

The efficacy of interleukin-1 (IL-1) inhibitors (anakinra, canakinumab) and interleukin-6 (IL-6) inhibitors (tocilizumab) as first-line biologic therapy for children with systemic juvenile idiopathic arthritis (sJIA) has been practically assured. However, a significant number of patients with sJIA discontinue biologic therapy due to lack of efficacy or safety concerns [1-3]. As a result, the patient has to be switched to another biologic. Based on real-world observational studies, $21-47 \%$ of patients with sJIA switch to a second, or subsequent, biologic disease-modifying antirheumatic drug (DMARD) [1-3], within 7-16 
months [2, 3]. According to the Russian Register of patients with sJIA (2002-2015; $n=384), 68 \%$ of patients received at least one dose of at least one biologic (most often tocilizumab), with every third patient (32\%) requiring a switch to a second or subsequent biologic DMARD [4].

Current guidelines developed by American College of Rheumatology ([ACR] 2013) [5] and National Health Service ([NHS] 2015) [6] state that a biologic with a different mechanism of action should be prescribed when considering a second or subsequent biologic in patients with SJIA who have continued disease activity with, or an intolerance to, first-line biologic therapy. National Russian guidelines (2021) contain similar recommendations [7]. To support this expert opinion, efficacy and safety studies of switch regimens in patients with SJIA are required (e.g., tocilizumab with a subsequent switch to canakinumab or any other dual regimen in line with current guidelines). In addition, when conducting such studies, it is important to consider the local/regional specificity of rheumatology care. Only two small-scale, openlabel extension studies have demonstrated high efficacy of switching from tocilizumab to canakinumab in patients with SJIA at Week 28 [8] (subsequently confirmed for 48 weeks [9]) and at Month 12 [10].

This study aimed to evaluate the 1-year efficacy and safety of canakinumab as a second-line biologic after tocilizumab treatment failure in children with SJIA in routine clinical practice.

\section{Methods}

\section{Study design}

This single-center retrospective cohort study evaluated treatment outcomes in children with SJIA who switched from tocilizumab to canakinumab. Data were collected for participants enrolled in one of two clinical trials at the study center, CACZ885G2301E1 (G2301; NCT00891046) and CACZ885G2306 (G2306; NCT02296424) [11], in addition to those who switched treatment in routine clinical practice.

\section{Setting}

The patients were those who had been hospitalized and followed up at the rheumatology department of the National Medical Research Centre for Children's Health in Moscow (previously known as the National Centre for Children's Health until November 2016 and then the National Scientific and Practical Centre for Children's Health, Moscow until July 2017) from August 2012 (start of canakinumab studies at the center) to July 2020 (uploading of the database with routinely collected health data to generate the study population).

\section{Participants (eligibility criteria)}

\section{Inclusion criteria}

Patients with SJIA aged 2 to 18 years who were prescribed to initiate canakinumab therapy over no more than 2 weeks after discontinuation of tocilizumab (received at least one dose of the drug) due to lack of 
efficacy or an adverse reaction were included in the analysis. The decision on the need to discontinue tocilizumab was made by the physician, as part of their routine clinical practice.

\section{Exclusion criteria}

Not planned.

\section{Exposure (canakinumab therapy)}

All patients (both from routine practice and participants of G2301 and G2306) were prescribed canakinumab, a human anti-IL-1 $\beta$ monoclonal antibody, at a dose of $4 \mathrm{mg} / \mathrm{kg}$ subcutaneously every 4 weeks, maximum $300 \mathrm{mg}$. In accordance with the protocol, dose tapering was applied for participants of G2306 providing that they achieved clinical remission (inactive disease for $\geq 24$ subsequent weeks) without the use of corticosteroids or methotrexate. Tapering regimens included either a dose reduction from $4 \mathrm{mg} / \mathrm{kg}$ to $2 \mathrm{mg} / \mathrm{kg}$ (no earlier than Week 28), and then to $1 \mathrm{mg} / \mathrm{kg}$ (no earlier than Week 52), or increased inter-injection intervals, followed by discontinuation. Dose tapering was not scheduled for participants of G2301 or those in routine clinical practice. Any changes to therapy were made in accordance with the physician's decision.

\section{Outcomes}

Classification of primary and secondary outcomes were not pre-defined for patients switching to canakinumab in the clinical practice setting.

\section{Primary efficacy outcomes}

The study endpoints were as follows: at least $30 \%$ improvement based on the pediatric criteria of ACR30 [12], the achievement of inactive disease according to the 71-point Juvenile Arthritis Disease Activity Score (JADAS-71 $=0$ points) [13] and clinical remission according to the criteria of ACR SJIA clinical inactive disease [14]. Follow-up for treatment outcomes was 12 months or the closest time, but not less than 6 and not more than 18 months from the day of the first dose of canakinumab.

\section{Secondary efficacy outcomes}

Clinical parameters: the percentage of patients with morning joint stiffness lasting for $>15-20$ minutes, active joints, systemic signs of disease activity (fever, rash, serositis, hepatomegaly/splenomegaly and/or lymphadenopathy).

Lab tests: erythrocyte sedimentation rate $(\mathrm{ESR})>20 \mathrm{~mm} / \mathrm{h}$, C-reactive protein (CRP) levels $>5 \mathrm{mg} / \mathrm{L}$.

Assessment criteria: nonzero values of disease activity as assessed by the treating physician using a 100-mm visual analogue scale (VAS), the patient's wellbeing assessed by the patient's parent using a 100-mm VAS and functional impairment based on the Childhood Health Assessment Questionnaire (CHAQ) [15] using the patient's parent estimate. 
Monitoring of the treatment outcomes using additional parameters was conducted within the same time frame as for those using primary endpoints of the study.

\section{Safety outcomes}

Safety of canakinumab-based therapy was assessed using the adverse event (AE) rates, i.e., any untoward medical events (unfavorable changes in the patient's health status or parameters reflecting the patient's health status) that occurred immediately after drug administration or during its use.

\section{Data sources}

Data on patients, administered treatment and its outcomes, were extracted from electronic outpatient medical records of the rheumatology department and medical record documentation generated in local healthcare facilities during the time between hospital admissions (discharge summaries, outpatient medical records and medical reports). The latter were provided to the treating physicians of the rheumatology department by the parents of patients with SJIA. The analysis of laboratory parameters of the disease activity was carried out in the clinical diagnostic laboratory at the National Medical Research Centre of Children's Health. Health data were accumulated in the 1C:Enterprise application (1C Company, Russia). After uploading the routinely collected health data database (tables with data of all study subjects), the data entered were checked to exclude input errors.

Treatment efficacy measures (primary and secondary), as well as safety measures, were determined using the data contained in electronic outpatient medical records. When assessing treatment safety, information from medical record documentation provided by the patients' parents (cases of AEs in the between admission period) was also considered.

\section{Statistical procedures}

\section{Sample size calculation}

The sample size was not pre-calculated.

\section{Statistical methods}

Statistical analysis was performed using SciStat, the online service by MedCalc Software (www.scistat.com). The description of quantitative values was presented as median (25th; 75th percentile). A Wilcoxon test was used to compare quantitative indicators in the dependent samples (before and after values). The event rates for the primary outcome measures were described indicating a 95\% confidence interval ( $\mathrm{Cl}$ ) without continuity correction (using online service www.vassarstats.net) and the rate per 100 person-years.

\section{Sensitivity analysis}


The choice of the dependent variable (achievement of clinical remission according to the criteria of ACR SJIA clinical inactive disease) was carried out after data collection. The ACR30 and JADAS-71 criteria as such were abandoned due to the small number of patients with lack of efficacy. Treatment outcomes were analyzed taking into account the effect of all baseline clinical, lab and assessment characteristics (at disease onset or before canakinumab treatment initiation). Univariate Cox proportional hazards regression analysis was used to evaluate the effects.

\section{Ethical review}

The protocols of G2301 and G2306 trials (dated 10 August 2012 and 5 December 2014, respectively) were approved by the local Ethics Committee of the National Medical Research Centre of Children's Health (at the time of the Ethics Committee opinion issue, referred to as National Centre for Children's Health). The inclusion of data from routine clinical practice in the study was not agreed with the Ethics Committee. At admission, the parents of all patients plus the patients themselves if aged $\geq 15$ years provided their written informed consent allowing for the use of examination and treatment results for scientific purposes.

\section{Results}

\section{Patient disposition}

During the study period, a total of 250 patients with SJIA who received tocilizumab were monitored in the rheumatology department, of whom $65(26 \%)$ discontinued therapy due to lack of efficacy or an adverse reaction. Tocilizumab was switched to canakinumab in 46 patients; 35 patients initiated canakinumab therapy in routine clinical practice, three upon entry to the G2301 trial and eight upon entry to the G2306 trial. The most common reason for tocilizumab withdrawal was secondary treatment failure (defined by physicians as failure to meet the criteria of ACR SJIA clinical inactive disease, occurring at any time following initial achievement of clinical remission following initial treatment effect; $n=32,70 \%) ; 12$ $(26 \%)$ patients withdrew due to an adverse reaction, and two (4\%) due to primary treatment failure (defined by physicians as failure to achieve ACR SJIA clinical inactive disease within the first 6 months after drug initiation).

The study outcomes were followed up in 45 patients (one patient did not attend the follow-up examination and treatment control in the period from Month 6 to Month 18 for an unknown reason).

\section{Baseline characteristics}

Patient demographics and disease characteristics are presented in Table 1. Approximately half of the patients (52\%) were female. the median age at the time of sJIA onset was 3.4 years and median age at the time of canakinumab treatment initiation being 8.2 years. Duration of sJIA before canakinumab initiation did not exceed 2 years in more than half (52\%) of the patients. Systemic manifestations (fever, rash, serositis, hepatomegaly/splenomegaly and/or lymphadenopathy) were observed in 42 (91\%) 
patients. At the time of canakinumab initiation, all patients received treatment with non-steroidal antiinflammatory drugs (NSAIDs), more than half received oral corticosteroids, more than $40 \%$ received methotrexate; and $17 \%$ received cyclosporine A. A total of $37(80 \%)$ patients received at least one nonbiologic DMARD (oral corticosteroids, methotrexate and/or cyclosporine A), of which 16 (43\%) patients received concurrently $\geq 2$ immunosuppressants.

\section{Primary efficacy outcomes}

Median follow-up for patients with known outcomes was 359 (282; 404) days; range, 184-476 days. Pediatric ACR30 criteria was achieved in 43 patients (96\%; 95\% Cl, 85-99), inactive disease based on JADAS-71 was achieved in 42 patients $(93 \%$; $95 \% \mathrm{Cl}, 82-98)$, and clinical remission in $37(82 \% ; 95 \% \mathrm{Cl}$, 69-91) of the 45 patients with follow-up data available. After adjustment for actual time-at-risk, the rates were 83,85 and 73 events per 100 person-years, respectively.

During follow-up, canakinumab was discontinued in one patient (discontinuation occurring after 14 months due to tuberculosis [TB] detection), the dose was reduced to $2 \mathrm{mg} / \mathrm{kg}$ per injection in three patients, and the interval between injections was increased up to 8 weeks in one patient. In addition, during the follow-up period, NSAIDs were administered in two (4\%) patients, oral corticosteroids in 20 (44\%), methotrexate in $12(27 \%)$ and cyclosporine A in nine (20\%) patients. A total of $27(60 \%)$ patients received at least one non-biologic DMARD (oral corticosteroids, methotrexate and/or cyclosporine A), of which 13 (48\%) patients received concurrently $\geq 2$ immunosuppressants.

\section{Secondary efficacy outcomes}

At the time of the follow-up examination (45 patients): morning stiffness persisted in three (7\%) patients; active joints in two patients (4\%), with two active joints in each case; systemic disease manifestations in three patients $(7 \%)$, with fever in two patients $(4 \%)$, hepatomegaly/splenomegaly and lymphadenopathy in one patient $(2 \%)$. At least one clinical sign of the active disease was noted in seven of $45(16 \%)$ patients.

At the end of follow-up, median ESR was $3(2 ; 7) \mathrm{mm} / \mathrm{h}$ (data for 44 patients presented) $(p<0.001$; hereinafter vs. the baseline), median CRP values $(n=45)$ were $1.0(1.0 ; 1.9) \mathrm{mg} / \mathrm{L}(p<0.001)$. The test parameters exceeding upper reference limits (ESR $>20 \mathrm{~mm} / \mathrm{h}, \mathrm{CRP}>5 \mathrm{mg} / \mathrm{L}$ ) were found in $4 / 44(9 \%)$ and $5 / 45(11 \%)$ patients. At least one laboratory sign of the disease was noted in $6 / 45(13 \%)$ patients.

Nonzero values of the disease activity assessment by the physician (100mm VAS) were in eight (18\%) of 45 patients; the median was $10 \mathrm{~mm}(9 ; 16)$; the range was 5 to $45 \mathrm{~mm}$. The parents of the same eight patients gave nonzero scores for their wellbeing; the median was $15 \mathrm{~mm}(10 ; 27)$; the range was 10 to 55 $\mathrm{mm}$. Nonzero scores of the parents' assessment of functional insufficiency according to CHAQ were reported in four (9\%) patients, with a range of 0.125 to 0.5 . Nonzero scores for at least one estimated parameter were identified in $8 / 45(18 \%)$ patients. 
In total, at the end of follow-up, any of the above clinical, laboratory and/or estimated parameters of disease activity (including parents' scores of wellbeing and functional insufficiency in pediatric patients) was observed in 11 (24\%) of 45 patients, of which five had only clinical, laboratory or estimated (indicated by the patient's physician or parent) sign of active disease.

\section{Safety analysis}

During follow-up, 23 AEs were reported in 19/45 (42\%) patients, of whom 11 had an acute respiratory or intestinal infection, four had leukopenia/neutropenia, four had elevated liver transaminase levels and four had TB infection (including one case of a positive M. tuberculosis test result). Electronic medical records of $5 / 45(11 \%)$ patients contain the history of macrophage activation syndrome (MAS) during the use of canakinumab, with a favorable outcome in all patients.

\section{Predictors of remission}

According to the criteria of ACR SJIA clinical inactive disease, none of the sample characteristics (see Table 1) were associated with achievement of clinical remission during follow-up.

\section{Discussion}

\section{Summary of primary study findings}

The majority of patients with sJIA treated with canakinumab after discontinuation of tocilizumab $(74 \%$ due to lack of efficacy, either primary or secondary) achieved clinical remission, inactive disease and/or improvements in disease signs within a year. In addition, the use of non-biologic DMARDs, such as oral corticosteroids, methotrexate and/or cyclosporine A, also decreased. The 'survival' of canakinumab therapy during follow-up was high, with only one case of therapy withdrawal due to TB infection. At least one $A E$ (most frequently infection) was reported in more than $40 \%$ of patients.

\section{Limitations}

\section{Representativeness of the study sample}

The study involved patients who were followed up at a single center only. In these cases, the patient management does not reflect all specific aspects of routine clinical practice in the study region and the extent to which these findings can be generalized to other healthcare systems is not known. Moreover, the rheumatology department, whose patients were enrolled in the study, is a subdivision of the largest Russian pediatric center, the capabilities of which (expert, diagnostic, therapeutic) can significantly exceed those of regional clinical centers. This is reflected by the enrolment of every fourth patient $(n=11$; 24\%) we analyzed in the international clinical trial (G2301 and G2306). In these trials, therapy is predetermined by the protocol and treatment control and disease outcomes monitoring are more stringent than in routine clinical practice. In addition, this study enrolled patients from the Russian Federation only; 
it is not known to what extent the findings can be generalized to patients in other countries with other healthcare systems.

\section{Small-scale sample}

One of the important advantages of large-scale cohort studies is the heterogeneity of its sample, helping to gain insight into the results of medical interventions in a setting as close to real-world clinical practice as possible. In small-scale trials, the description of relatively rare treatment outcomes (both favorable and unfavorable, including AEs) is obviously impossible.

\section{Variability of follow-up period}

Many of our pediatric patients were from remote regions of Russia, which limited their ability to attend regular follow-ups. Attending the clinical center for at least one assessment per year was recommended for all patients, but some were unable to do this routinely. As a result, the spread of the actual follow-up values varied from Month 6 to Month 16. Although the median period was 12 months, the distribution of the follow-up duration in the study sample was shifted to the left (coefficient of skewness -0.271 ). This limits the interpretation of the study results.

\section{Routinely collected health data}

We analyzed the data obtained from electronic outpatient medical records. The quality of such data may give rise to reasonable doubts [16], particularly with regards to safety information. Furthermore, missing data is often a limitation for the use of medical records. In this study, there were only isolated cases of missing data, which, in our opinion, did not significantly affect the results obtained.

\section{No control group}

We cannot directly attribute the effects of therapy (both beneficial and adverse) solely to the effect of canakinumab, since the majority of patients ( $80 \%$ at baseline and $60 \%$ at follow-up) received at least one non-biologic DMARD, such as oral corticosteroids, methotrexate and/or cyclosporine A. Similarly, it should be noted that approximately three-quarters of patients were prescribed canakinumab in view of lack of efficacy of tocilizumab (mainly secondary failures) and at the same time, while on canakinumab, a decrease in the proportion of patients receiving oral corticosteroids (from $57 \%$ to $44 \%$ ) and methotrexate (from $43 \%$ up to $27 \%$ ) was reported. The results described with canakinumab treatment were therefore achieved against a less intense background regimen of non-biologic DMARDs. Moreover, this does not take into account a change in the dose of non-biologic DMARDs, the reduction of which has been shown in randomized clinical trials with canakinumab $[9,17]$.

\section{Interpretation Of Study Results}

\section{Efficacy of switching from tocilizumab to canakinumab}


In our study, the minimum significant improvement according to the pediatric ACR30 criteria was reported in $96 \%$ (43/45) patients (median follow-up of 12 months or 359 days) or in $85 \%$ in terms of the rate per 100 person-years. Similar results were demonstrated in clinical trials by Brunner et al. 2015 [10] and Nishimura et al. 2021 [9]. In the former study, after switching from tocilizumab (withdrawn due to lack of efficacy or safety) to canakinumab, the ACR30 criteria was achieved in 23/24 (96\%) patients with sJIA at Month 12, in the latter, in 16/19 (84\%) at approx. Month 11 (median 337 days). A similar result (ACR30 in $86 \%$ at the median follow-up of 8 months or 236 days) was obtained in a clinical trial (withdrawal phase) by Ruperto et al. 2012, where $80 \%$ of patients received tocilizumab before canakinumab [17]. Moreover, in the above-mentioned trials, roughly one third of patients discontinued glucocorticoids following initiation of canakinumab [9,17]; in our study, oral glucocorticoids were discontinued in six of the 26 patients $(23 \%)$ who were receiving these drugs at the time of administration of the first dose of canakinumab.

Several hypotheses can explain the lack of efficacy of tocilizumab in patients with sJIA and, accordingly, the efficacy of switching to canakinumab. First, IL-1 but not IL-6, can be considered a primary mediator of autoimmune (in fact, aseptic) inflammation. In particular, in vitro studies demonstrate that SJIA is an IL$1 \beta$-mediated disease [18]. The pathogenetic role of these cytokines in the development of autoimmune processes and arthritis was confirmed both in IL-1Ra [21] and IL-6-deficient mice [22] as well as the initial clinical studies of IL-1- [18] and IL-6-inhibitors [23]. However, it is noteworthy that systemic inflammation in combination with arthritis is observed in IL-1-overexpressing [24], but not IL-6-overexpressing transgenic mice $[25,26]$. In addition, the aseptic inflammation model showed that IL-6 is not detectable in the blood serum of IL-1 $\beta$-deficient mice [27], while, on the contrary, IL-1 is induced normally in IL-6deficient mice [28]. It was also found that in mice transgenic for IL-1a (activation of IL-1 signaling) and prone to arthritis, IL- 6 deficiency resulting from knockout of the corresponding gene reduces, but does not cancel, pathological changes in the joints [24]. Finally, in newly diagnosed untreated patients with sJIA, high expression levels of genes involved in the negative regulation of IL-1R signaling have been observed [29].

Secondly, different sensitivity to tocilizumab in patients with sJIA may be fundamental. For instance, it has been shown that the lack of efficacy of tocilizumab is associated with initially high concentrations of IL-6 [30, 31], sIL-6R [32, 33] and a low concentration of soluble gp130 [31], which is a natural inhibitor of the IL-6 trans-signaling pathway [34]. The effect of high levels of IL- 6 may be due to the competition of significant amounts of cytokine with tocilizumab for binding sites (IL-6R) on the membranes of target cells, i.e., immunocompetent cells and hepatocytes, but not for soluble forms of the receptor, which is confirmed in studies with IL-6-dependent myeloma cell line and recombinant BaF/IL-6R [35]. It can be assumed that in some patients the recommended dose of tocilizumab may not be enough to suppress both a high concentration of soluble IL-6R and significant amounts of the IL-6/sIL-6R complex due to a low concentration of soluble gp130.

Thirdly, there are rare cases of treatment failure and/or hypersensitivity reactions associated with antibodies to biologics and, in particular, to tocilizumab [36]. In these cases, the efficacy of IL-1 inhibitors can be expected as it can be considered as a therapy for 'biologically naïve' patients. The development of 
antibodies to canakinumab in such patients is unlikely as no anti-drug neutralizing antibodies were detected [11, 37].

Finally, tocilizumab does not affect the concentration of IL-1 [30], i.e., does not have an indirect effect on the key mechanism of SJIA pathogenesis. In this regard, it is important to note that high baseline levels of IL-1 in serum is associated with a lower efficacy of tocilizumab after 16 [31] and 52 weeks [38] of therapy. In contrast, down-modulation of IL- 6 has been reported as a result of therapy with IL-1 inhibitors, occurring as early as 1 week after anakinra initiation [39] and 3 days after the administration of canakinumab [40]. Down-modulation of IL-6 in patients with sJIA on canakinumab therapy (switching from tocilizumab) has been shown to persist for 24 and 48 weeks [9].

Therefore, the benefits of switching from an IL- 6 inhibitor to an IL-1 inhibitor and, in particular, from tocilizumab to canakinumab can potentially be explained by: IL-1-mediated links in the pathogenesis of SJIA; unequal sensitivity to tocilizumab in patients and the effect exerted by IL-1 inhibitors on the SJIA mechanisms associated both with IL-1 and IL-6.

\section{Safety of switching from tocilizumab to canakinumab}

In our study, AEs were reported in more than $40 \%$ of patients. According to the findings from clinical studies in patients with SJIA who received canakinumab after tocilizumab, AEs were reported in 78-100\% of patients $[9,17]$. This difference can be explained by the study design and, in particular, by the use of electronic medical records to estimate the AEs. There is no doubt that routinely collected health data include only the most remarkable deviations in the health status, which, in the physicians' opinion, are obviously related to the prognosis. In this regard, our data on the frequency of AEs are consistent with the rates of serious AEs reported in the study by Nishimura et al. (42\%) [9]. According to the German BiKeR Registry, the rate of serious AEs with canakinumab was even lower, 20 cases per 100 patient-years [41].

It should be separately noted that in our study, $11 \%$ of patients developed MAS while using canakinumab and that this is higher than the rate observed in clinical trials of canakinumab (5.3\% or 2.8 per 100 patient-years) [42] where the incidence rate of (probable) MAS observed in canakinumab-treated patients was comparable to the incidence reported in patients in the placebo group. In part, the higher incidence of MAS in our study may be due to the limited diagnostic accuracy of the criteria for this condition (overdiagnosis) [43]. Indeed, in small-scale studies (with < 100 subjects $[29,44,45]$ ) the frequency of reported cases of MAS significantly exceeds that reported in large-scale studies (> 100 patients [46-48]) (12-20\% vs. $4-7 \%$, respectively).

\section{Conclusions}

Short-term (6-18 months, 12 months on average) canakinumab therapy was found to be potentially effective as second-line biologic therapy after discontinuation of tocilizumab in patients with SJIA. This was achieved along with a decrease in the use of non-biologic DMARDs, such as oral corticosteroids, and/or cyclosporine Amethotrexate. The 'survival' of canakinumab therapy during the follow-up was high, 
with only one case of drug withdrawal. At least one AE (most frequently, infections) was reported in more than $40 \%$ of patients.

\section{Abbreviations}

ACR: American College of Rheumatology; AE: Adverse event; CHAQ: Childhood Health Assessment Questionnaire; Cl: Confidence interval; CRP: C-reactive protein; DMARD: disease-modifying antirheumatic drugs; ESR: Erythrocyte sedimentation rate; IL: Interleukin; JADAS: Juvenile Arthritis Disease Activity Score; MAS: Macrophage activation syndrome; NHS: National Health Service; NSAID: Non-steroidal antiinflammatory drug; sJIA: Systemic juvenile idiopathic arthritis; TB: tuberculosis; VAS: Visual analogue scale

\section{Declarations}

\section{Ethics approval and consent to participate}

The protocols of G2301 and G2306 trials (dated 10 August 2012 and 5 December 2014, respectively) were approved by the local Ethics Committee of the National Medical Research Centre of Children's Health (at the time of the Ethics Committee opinion issue, referred to as National Centre for Children's Health). The inclusion of data from routine clinical practice in the study was not agreed with the Ethics Committee. At admission, the parents of all patients plus patients themselves if aged $\geq 15$ years provided their written informed consent allowing for the use of examination and treatment results for scientific purposes.

\section{Consent for publication}

Not applicable.

\section{Availability of data and materials}

The datasets used and/or analyzed during the current study are available from the corresponding authors on reasonable request.

\section{Competing interests}

EA has received grant/research support from Roche, Pfizer, Centocor, Eli Lilly, and Novartis, and has participated in Speakers bureau sessions for Roche, AbbVie, Bristol-Myers, Squibb, MSD, Novartis and Pfizer.

TD has received grant/research support from Roche, Pfizer, Centocor, Eli Lilly, and Novartis, and has participated in Speakers bureau sessions for Roche, AbbVie, Bristol-Myers, Squibb, MSD, Novartis and Pfizer. 
KI has received grant/research support from Roche, Novartis and Sanofi.

OL has received grant/research support from Pfizer, Eli Lilly.

RD has received grant/research support from Roche, Pfizer, Centocor, Sanofi and Novartis, and has participated in Speakers bureau sessions for Roche, AbbVie, MSD, Novartis.

AM has received grant/research support from Eli Lilly has participated in Speakers bureau sessions for Novartis.

AF has received grant/research support from Amgen.

EK has participated in Speakers bureau sessions for Novartis.

IK has participated in Speakers bureau sessions for Novartis.

RS payment for lectures, presentations, and educational events for Eco-Vector LLC.

$A C, E C, M G$, and DV confirms that they have no competing interests to declare.

\section{Funding}

Funding for medical writing support in the development of the manuscript was provided by Novartis Pharma AG.

\section{Authors' contributions}

All authors read and approved the final manuscript. CRediT author statement: 


\begin{tabular}{|lll|}
\hline$\#$ & ROLE & AUTHORS \\
\hline 1 & Conceptualization & EA, RS \\
\hline 2 & Data curation & RS \\
3 & Formal analysis & RS \\
4 & Funding acquisition & EA \\
\hline 5 & Investigation & TD, KI, AC, EC, OL, RD, AM, AF, MG, DV \\
6 & Methodology & RS, EA \\
\hline 7 & Project administration & EA \\
\hline 8 & Resources & \\
\hline 9 & Software & \\
\hline 10 & Supervision & EA \\
\hline 11 & Validation & \\
\hline 12 & Visualization & EA, EK, RS \\
\hline 13 & Writing - original draft & RS, EA, EK \\
\hline 14 & Writing - review \& editing & \\
\hline
\end{tabular}

\section{Acknowledgements}

Medical writing support, under the direction of the authors, was provided by Sarah Johnston and Natalie Roberts of Ashfield MedComms, an Ashfield Health company.

\section{Authors' information (optional)}

\section{References}

1. Otten MH, Prince FH, Anink J, Ten Cate R, Hoppenreijs EP, Armbrust W, et al. Effectiveness and safety of a second and third biological agent after failing etanercept in juvenile idiopathic arthritis: results from the Dutch National ABC Register. Ann Rheum Dis. 2013;72(5):721-7. doi:

10.1136/annrheumdis-2011-201060.

2. Woerner A, Uettwiller F, Melki I, Mouy R, Wouters C, Bader-Meunier B, Quartier P. Biological treatment in systemic juvenile idiopathic arthritis: achievement of inactive disease or clinical remission on a first, second or third biological agent. RMD Open. 2015;1(1):e000036. doi: 10.1136/rmdopen-2014000036.

3. Kearsley-Fleet L, Heaf E, Davies R, Baildam E, Beresford MW, Foster HE, et al. Frequency of biologic switching and the outcomes of switching in children and young people with juvenile idiopathic 
arthritis: a national cohort study. Lancet Rheumatol. 2020;2(4):e217-26. doi: 10.1016/S26659913(20)30025-4.

4. Alekseeva El, Lomakina OL, Valieva SI, Bzarova TM, Nikishina IP, Zholobova YS, et al. Features of the drug therapy of children with systemic juvenile idiopathic arthritis: analysis results of the All-Russian Register of the Union of Pediatricians of Russia. Voprosy Sovremennoi Pediatrii - Current Pediatrics. 2016;15(1):59-67. doi: 10.15690/vsp.v15i1.1500.

5. Ringold S, Weiss PF, Beukelman T, DeWitt EM, llowite NT, Kimura Y, et al. 2013 update of the 2011 American College of Rheumatology recommendations for the treatment of juvenile idiopathic arthritis: recommendations for the medical therapy of children with systemic juvenile idiopathic arthritis and tuberculosis screening among children receiving biologic medications. Arthritis Rheum. 2013;65(10):2499-512. doi: 10.1002/art.38092.

6. NHS England. Clinical commissioning policy statement: biologic therapies for the treatment of juvenile idiopathic arthritis. 2015.

https://www.england.nhs.uk/commissioning/wp-content/uploads/sites/ 12/2015/10/e03pd-bio-therapies-jia-oct15.pdf. Accessed 25 March 2021

7. Systemic-onset juvenile arthritis. Clinical guidelines, 2021. https://aspirrerussia.ru/upload/medialibrary/e44/КР\%20cЮИА_АДР.pdf. Accessed 26 May 2021.

8. Takei S, Hara R, Umebayashi H, Iwata N, Imagawa T, Shimizu M, et al. THU0599 Evaluation of efficacy and safety of canakinumab in Japanese patients with systemic juvenile idiopathic arthritis in phase iii clinical trial, composed predominantly of patients with prior use of tocilizumab. Ann Rheum Dis. 2018;77:499-500. doi: 10.1136/annrheumdis-2018-eular.2464

9. Nishimura K, Hara R, Umebayashi H, Takei S, Iwata N, Imagawa T, et al. Efficacy and safety of canakinumab in systemic juvenile idiopathic arthritis: 48-week results from an open-label phase III study in Japanese patients. Mod Rheumatol. 2021;31(1):226-34. doi:

10.1080/14397595.2020.1783163.

10. Brunner HI, Ruperto N, Quartier P, Constantin T, Berkun Y, Calvo-Penedes I, et al. Efficacy of canakinumab in systemic juvenile idiopathic arthritis patients previously exposed to biologics [abstract]. Arthritis Rheumatol. 2015;67(Suppl 10). https://acrabstracts.org/abstract/efficacy-ofcanakinumab-in-systemic-juvenile-idiopathic-arthritis-patients-previously-exposed-to-biologics/ Accessed 23 March 2021.

11. Quartier P, Alexeeva E, Constantin T, Chasnyk V, Wulffraat N, Palmblad K, et al. Tapering canakinumab monotherapy in patients with systemic juvenile idiopathic arthritis in clinical remission: results from a Phase IIIb/IV open-label, randomized study. Arthritis Rheumatol. 2021;73(2):336-46. doi: 10.1002/art.41488

12. Giannini EH, Ruperto N, Ravelli A, Lovell DJ, Felson DT, Martini A. Preliminary definition of improvement in juvenile arthritis. Arthritis Rheum. 1997;40(7):1202-9. doi: 10.1002/art.1780400703

13. Consolaro A, Negro G, Chiara Gallo M, Bracciolini G, Ferrari C, Schiappapietra B, et al. Defining criteria for disease activity states in nonsystemic juvenile idiopathic arthritis based on a three-variable 
juvenile arthritis disease activity score. Arthritis Care Res (Hoboken). 2014;66(11):1703-9. doi: 10.1002/acr.22393.

14. Wallace CA, Giannini EH, Huang B, Itert L, Ruperto N; Childhood Arthritis Rheumatology Research Alliance; Pediatric Rheumatology Collaborative Study Group; Paediatric Rheumatology International Trials Organisation. American College of Rheumatology provisional criteria for defining clinical inactive disease in select categories of juvenile idiopathic arthritis. Arthritis Care Res (Hoboken). 2011;63(7):929-36. doi: 10.1002/acr.20497.

15. Ruperto N, Ravelli A, Pistorio A, Malattia C, Cavuto S, Gado-West L, et al. Cross-cultural adaptation and psychometric evaluation of the Childhood Health Assessment Questionnaire (CHAQ) and the Child Health Questionnaire (CHQ) in 32 countries. Review of the general methodology. Clin Exp Rheumatol. 2001;19(4 Suppl 23):S1-9.

16. Langan SM, Schmidt SA, Wing K, Ehrenstein V, Nicholls SG, Filion KB et al. The reporting of studies conducted using observational routinely collected health data statement for pharmacoepidemiology (RECORD-PE) Br Med J. 2018;363 :k3532 doi:10.1136/bmj.k3532.

17. Ruperto N, Brunner HI, Quartier P, Constantin T, Wulffraat N, Horneff G, et al. Two randomized trials of canakinumab in systemic juvenile idiopathic arthritis. N Engl J Med. 2012;367(25):2396-406. doi: 10.1056/NEJMoa1205099.

18. Pascual V, Allantaz F, Arce E, Punaro M, Banchereau J. Role of interleukin-1 (IL-1) in the pathogenesis of systemic onset juvenile idiopathic arthritis and clinical response to IL-1 blockade. J Exp Med. 2005;201:1479-86. doi: 10.1084/jem.20050473

19. de Benedetti F, Massa M, Robbioni P, Ravelli A, Burgio GR, Martini A. Correlation of serum interleukin6 levels with joint involvement and thrombocytosis in systemic juvenile rheumatoid arthritis. Arthritis Rheum. 1991;34(9):1158-63. doi: 10.1002/art.1780340912.

20. Vilaiyuk S, Lerkvaleekul B, Soponkanaporn S, Setthaudom C, Buranapraditkun S. Correlations between serum interleukin 6 , serum soluble interleukin 6 receptor, and disease activity in systemic juvenile idiopathic arthritis patients treated with or without tocilizumab. Cent Eur J Immunol. 2019;44(2):150-8. doi: 10.5114/ceji.2019.87066.

21. Horai R, Saijo S, Tanioka H, Nakae S, Sudo K, Okahara A, et al. Development of chronic inflammatory arthropathy resembling rheumatoid arthritis in interleukin 1 receptor antagonist-deficient mice. J Exp Med. 2000;191(2):313-20. doi: 10.1084/jem.191.2.313.

22. Ohshima S, Saeki Y, Mima T, Sasai M, Nishioka K, Nomura S, et al. Interleukin 6 plays a key role in the development of antigen-induced arthritis. Proc Natl Acad Sci USA. 1998;95(14):8222-6. doi: 10.1073/pnas.95.14.8222.

23. Yokota S, Miyamae T, Imagawa T, Iwata N, Katakura S, Mori M, et al. Therapeutic efficacy of humanized recombinant anti-interleukin-6 receptor antibody in children with systemic-onset juvenile idiopathic arthritis. Arthritis Rheum. 2005;52(3):818-25.

24. Oike T, Kanagawa H. Sato Y, Kobayashi T, Nakatsukasa H, Miyamoto K, et al. IL-6, IL-17 and Stat3 are required for auto-inflammatory syndrome development in mouse. Sci Rep. 2018;8:15783. doi: 
10.1038/s41598-018-34173-5.

25. Suematsu S, Matsuda T, Aozasa K, Akira S, Nakano N, Ohno S, et al. IgG1 plasmacytosis in interleukin 6 transgenic mice. Proc Natl Acad Sci USA. 1989;86(19):7547-51. doi:

10.1073/pnas.86.19.7547.

26. De Benedetti F, Rucci N, Del Fattore A, Peruzzi B, Paro R, Longo M, et al. Impaired skeletal development in interleukin-6-transgenic mice: a model for the impact of chronic inflammation on the growing skeletal system. Arthritis Rheum. 2006;54(11):3551-63. doi: 10.1002/art.22175.

27. Fantuzzi G, Dinarello CA. The inflammatory response in interleukin-1 beta-deficient mice: comparison with other cytokine-related knock-out mice. J Leukoc Biol. 1996;59(4):489-93. doi:

10.1002/jlb.59.4.489.

28. Fattori E, Cappelletti M, Costa P, Sellitto C, Cantoni L, Carelli M, et al. Defective inflammatory response in interleukin 6-deficient mice. J Exp Med. 1994;180(4):1243-50. doi: 10.1084/jem.180.4.1243.

29. Fall N, Barnes M, Thornton S, Luyrink L, Olson J, llowite NT, et al. Gene expression profiling of peripheral blood from patients with untreated new-onset systemic juvenile idiopathic arthritis reveals molecular heterogeneity that may predict macrophage activation syndrome. Arthritis Rheum. 2007;56(11):3793-804. doi: 10.1002/art.

30. Shimamoto K, Ito T, Ozaki Y, Amuro H, Tanaka A, Nishizawa T, et al. Serum interleukin 6 before and after therapy with tocilizumab is a principal biomarker in patients with rheumatoid arthritis. J Rheumatol. 2013;40(7):1074-81. doi: 10.3899/jrheum.121389.

31. Uno K, Yoshizaki K, Iwahashi M, Yamana J, Yamana S, Tanigawa M, Yagi K. Pretreatment prediction of individual rheumatoid arthritis patients' response to anti-cytokine therapy using serum cytokine/chemokine/soluble receptor biomarkers. PloS one 2015;10(7):e0132055. doi: 10.1371/journal.pone.0132055

32. Diaz-Torne C, Ortiz MDA, Moya P, Hernandez MV, Reina D, Castellvi I, et al. The combination of IL-6 and its soluble receptor is associated with the response of rheumatoid arthritis patients to tocilizumab. Semin Arthritis Rheum. 2018;47(6):757-64. doi: 10.1016/j.semarthrit.2017.10.022

33. Nishina N, Kikuchi J, Hashizume M, Yoshimoto K, Kameda H, Takeuchi T. Baseline levels of soluble interleukin-6 receptor predict clinical remission in patients with rheumatoid arthritis treated with tocilizumab: implications for molecular targeted therapy. Ann Rheum Dis. 2014;73(5):945-7. doi:10.1136/annrheumdis-2013-204137

34. Jostock T, Müllberg J, Ozbek S, Atreya R, Blinn G, Voltz N, et al. Soluble gp130 is the natural inhibitor of soluble interleukin-6 receptor transsignaling responses. Eur J Biochem. 2001;268(1):160-7. doi: 10.1046/j.1432-1327.2001.01867.x.

35. Mihara M, Kasutani K, Okazaki M, Nakamura A, Kawai S, Sugimoto M, et al. Tocilizumab inhibits signal transduction mediated by both mIL-6R and sIL-6R, but not by the receptors of other members of IL-6 cytokine family. Int Immunopharmacol. 2005;5(12):1731-40. doi: 10.1016/j.intimp.2005.05.010. 
36. Doeleman MJH, van Maarseveen EM, Swart JF. Immunogenicity of biologic agents in juvenile idiopathic arthritis: a systematic review and meta-analysis. Rheumatology (Oxford). 2019;58(10):1839-49. doi: 10.1093/rheumatology/kez030.

37. Sun H, Van LM, Floch D, Jiang X, Klein UR, Abrams K, Sunkara G. Pharmacokinetics and pharmacodynamics of canakinumab in patients with systemic juvenile idiopathic arthritis. J Clin Pharmacol. 2016;56(12):1516-27. doi: 10.1002/jcph.754.

38. Okano T, Inui K, Tada M, Sugioka Y, Mamoto K, Wakitani S, et al. Levels of interleukin-1 beta can predict response to tocilizumab therapy in rheumatoid arthritis: the PETITE (predictors of effectiveness of tocilizumab therapy) study. Rheumatol Int. 2016;36(3):349-57. doi: 10.1007/s00296-015-3379-x

39. Gattorno M, Piccini A, Lasigliè D, Tassi S, Brisca G, Carta S, et al. The pattern of response to antiinterleukin-1 treatment distinguishes two subsets of patients with systemic-onset juvenile idiopathic arthritis. Arthritis Rheum. 2008;58(5):1505-15. doi: 10.1002/art.23437.

40. Brachat AH, Grom AA, Wulffraat N, Brunner HI, Quartier P, Brik R, et al. Early changes in gene expression and inflammatory proteins in systemic juvenile idiopathic arthritis patients on canakinumab therapy. Arthritis Res Ther 2017;19:13. doi: 10.1186/s13075-016-1212-x.

41. Klein A, Klotsche J, Hügle B, Minden K, Hospach A, Weller-Heinemann F, et al. Long-term surveillance of biologic therapies in systemic-onset juvenile idiopathic arthritis: data from the German BIKER registry. Rheumatology (Oxford). 2020;59(9):2287-98. doi: 10.1093/rheumatology/kez577.

42. Grom AA, llowite NT, Pascual V, Brunner HI, Martini A, Lovell D, et al. Rate and clinical presentation of macrophage activation syndrome in patients with systemic juvenile idiopathic arthritis treated with canakinumab. Arthritis Rheumatol. 2016;68(1):218-28. doi: 10.1002/art.39407.

43. Schulert GS, Minoia F, Bohnsack J, Cron RQ, Hashad S, KonÉ-Paut I, et al. Effect of biologic therapy on clinical and laboratory features of macrophage activation syndrome associated with systemic juvenile idiopathic arthritis. Arthritis Care Res (Hoboken). 2018;70(3):409-19. doi:

10.1002/acr.23277.

44. Behrens EM, Beukelman T, Paessler M, Cron RQ. Occult macrophage activation syndrome in patients with systemic juvenile idiopathic arthritis. J Rheumatol. 2007;34(5):1133-8.

45. Shimizu M, Nakagishi Y, Inoue N, Mizuta M, Ko G, Saikawa Y, et al. Interleukin-18 for predicting the development of macrophage activation syndrome in systemic juvenile idiopathic arthritis. Clin Immunol. 2015;160(2):277-81. doi: 10.1016/j.clim.2015.06.005.

46. Sawhney S, Woo P, Murray KJ. Macrophage activation syndrome: a potentially fatal complication of rheumatic disorders. Arch Dis Child. 2001;85(5):421-6. doi: 10.1136/adc.85.5.421.

47. Yokota S, Itoh Y, Morio T, Sumitomo N, Daimaru K, Minota S. Macrophage activation syndrome in patients with systemic juvenile idiopathic arthritis under treatment with tocilizumab. J Rheumatol. 2015;42(4):712-22. doi: 10.3899/jrheum.140288.

48. Moussa T, Abdelhak M, Edens C. How do pediatric rheumatologists diagnose macrophage activation syndrome in systemic onset juvenile idiopathic arthritis? An examination of the CARRA registry 
[abstract]. Arthritis Rheumatol. 2020;72(Suppl 4). https://acrabstracts.org/abstract/how-do-pediatricrheumatologists-diagnose-macrophage-activation-syndrome-in-systemic-onset-juvenile-idiopathicarthritis-an-examination-of-the-carra-registry/. Accessed 24 March 2021.

\section{Tables}

Table 1 Patient characteristics at baseline (canakinumab treatment initiation) 


\begin{tabular}{|c|c|}
\hline Variables & Values $(n=46)$ \\
\hline \multicolumn{2}{|l|}{ General characteristics } \\
\hline Female, $n(\%)$ & $24(52)$ \\
\hline Age at baseline*, years & $8.2(4.0 ; 12.9)$ \\
\hline Age at disease onset, years & $3.4(2.4 ; 5.5)$ \\
\hline Duration of SJIA*, years & $1.8(0.8 ; 5.8)$ \\
\hline - < 2 years (early arthritis), $n(\%)$ & $24(52)$ \\
\hline \multicolumn{2}{|l|}{ Clinical characteristics at baseline* } \\
\hline \multirow{6}{*}{$\begin{array}{l}\text { Systemic manifestations, } n(\%) \text { : } \\
\text { - fever } \\
\text { - rash } \\
\text { - hepatomegaly/splenomegaly } \\
\text { - lymphadenopathy } \\
\text { - serositis (pleuritis, pericarditis) }\end{array}$} & \\
\hline & $35(76)$ \\
\hline & $33(72)$ \\
\hline & $26(57)$ \\
\hline & $17(37)$ \\
\hline & $6(13)$ \\
\hline Macrophage activation syndrome, $n(\%)$ & $11(24)$ \\
\hline Active joint, $n(\%)$ & $34(74)$ \\
\hline - active joint count $(n=34)$ & $5(2 ; 8)$ \\
\hline $\mathrm{ESR}, \mathrm{mm} / \mathrm{h}$ & $26(4 ; 54)$ \\
\hline - > $20 \mathrm{~mm} / \mathrm{h}, n(\%)$ & $28(61)$ \\
\hline CRP, $\mathrm{mg} / \mathrm{L}^{\star \star}$ & $13(1 ; 62)$ \\
\hline - $>5 \mathrm{mg} / \mathrm{L}, n(\%)$ & $31(67)$ \\
\hline Physician's assessment of disease activity, 0-100 mm VAS & $45(34 ; 74)$ \\
\hline Parent's assessment of overall well-being, $0-100 \mathrm{~mm}$ VAS & $58(45 ; 81.5)$ \\
\hline CHAQ score ${ }^{\star \star \star}$ & $0.25(0.03 ; 0.88)$ \\
\hline JADAS-71 & $15.5(10 ; 24)$ \\
\hline \multicolumn{2}{|l|}{ Treatment at baseline } \\
\hline NSAIDs, $n(\%)$ & $46(100)$ \\
\hline Oral corticosteroids, $n(\%)$ & $26(57)$ \\
\hline Methotrexate, $n(\%)$ & $20(43)$ \\
\hline
\end{tabular}


Variables

Cyclosporine A, $n(\%)$
Values $(n=46)$

$8(17)$

NOTE:

Quantitative variables are presented as median (25th; 75th percentile).

${ }^{*}$ On the day of canakinumab initiation; ${ }^{*} \mathrm{CRP}$ values below the analytical sensitivity threshold of the measurement method ( $<1 \mathrm{mg} / \mathrm{L}$ ) were coded as equal to 1 (in 13 patients); ***Data from $12(26 \%)$ patients with zero values of the parameter were disregarded.

$C H A Q$ childhood health assessment questionnaire; CRP C-reactive protein; ESR erythrocyte sedimentation rate; JADAS Juvenile Arthritis Disease Activity Score; sJIA systemic juvenile idiopathic arthritis; VAS visual analogue scale; NSAID non-steroidal anti-inflammatory drug. 